\title{
The jump of reverse drift rate of V-shaped structure and the large-scale inhomogeneity of plasma density in the solar atmosphere
}

\author{
F. Y. $\mathrm{Xu}^{1,2}$ and H. A. $\mathrm{Wu}^{1,2}$ \\ 1 Purple Mountain Observatory, Chinese Academy of Sciences, Nanjing 210008, PR China \\ e-mail: pmoxfy@163.com \\ 2 National Astronomical Observatories, Chinese Academy of Sciences, Beijing 100012, PR China
}

Received 16 October 2002 / Accepted 31 March 2004

\begin{abstract}
A rare solar radio V-shaped structure was observed on August 25, 1999 by the $4.5-7.5 \mathrm{GHz}$ spectrograph of the Purple Mountain Observatory, China. It consists of a reverse drift component followed by a normal drift one, which seems to be an unusual frequency drift event because of the jump of reverse drift rate from 3.22 to $1.53 \mathrm{GHz} / \mathrm{s}$ and the fastest drift rate of $-3.53 \mathrm{GHz} / \mathrm{s}$. According to their different drift rates, the V-shaped structure can be divided into three parts. By the consideration of plasma emission of an electron beam, a three-component solar atmospheric model with large and small scale lengths $\lambda, \lambda_{1}$ and $\lambda_{2}$ to describe the equilibrium solar atmosphere and local inhomogeneity as well as the energy loss of the electron beam due to electron-electron collision is proposed. The local scale lengths $\lambda_{1}=5.5 \times 10^{9}, \lambda_{2}=7.7 \times 10^{9} \mathrm{~cm}$ and $\bar{v}_{1}=0.41 \mathrm{c}, \bar{v}_{2}=0.25 \mathrm{c}$ at Parts 1 and 2 of the reverse drift component are obtained. The variations of scale length of $\lambda_{1}<\lambda_{2}$ and $\bar{v}_{1}>\bar{v}_{2}$, especially $\bar{v}_{1}>\bar{v}_{2}$, could be responsible for the jump of drift rate. The normal drift rate would be interpreted as the increase of average energy $E^{\prime}$ of the electron beam at the mirror due to the decrease in electrons of lower energy via energy loss of collision. The three-component solar atmospheric model and the energy loss of the electron beam are discussed.
\end{abstract}

Key words. sun: flares - Sun: radio radiation

\section{Introduction}

Earlier observations of solar radio type III bursts were made mainly in meter/decimeter wavebands, and their properties were characterized by narrow bandwidth, frequency drift and harmonic structure etc. (Suzuki \& Dulk 1985). The characters of narrow bandwidth and frequency drift suggest that they may originate from a coherent emission mechanism such as from local plasma emission at its characteristic frequency, $\omega_{\mathrm{p}}$, excited by a source moving upward/downward along the field line. The exciting Langmuir wave would be converted into fundamental or harmonic escaping radiation at characteristic frequencies $\omega=\omega_{\mathrm{p}}, \omega=2 \omega_{\mathrm{p}}$ by scattering or mode-mode coupling processes (Zheleznyakov 1970; Melorose 1980). The frequency of escaping radiation follows the variation of exciting local plasma frequency which decreases/increases with time as the electron beam moves upward/downward, and leads to a detectable normal/reverse drift type III burst. Such a picture is still accepted today for type III or type III-like bursts.

Since the 1980s, the surveys of solar radio type III bursts have been conducted in microwave bands, such as $0.1-3 \mathrm{GHz}$, 4-8.5 GHz and 6-8.5 GHz (Stähli \& Benz 1987; Alloart et al. 1999; Bruggmann et al. 1990). The observed characters of microwave type III $\left(\mathrm{III}_{\mu}\right)$ bursts are similar to those of meter/decimeter type III bursts except that the drift rate of type III $_{\mu}$ bursts is almost two orders of magnitude faster than that of meter/decimeter type III bursts, and also the dominant drift direction is a reverse drift in the microwave band but normal one in the meter and decimeter waveband. What electron density is required for a type $\mathrm{III}_{\mu}$ burst? For example, in the range of $4.5-7.5 \mathrm{GHz}$, the required electron density is about $6 \times 10^{10}$ to $2 \times 10^{11} / \mathrm{cm}^{3}$ if harmonic emission is assumed. This probably corresponds to the region between the lower corona and higher chromosphere. Because type III and type III-like structures may be the reflection of the electron beam trace or magnetic field configuration, the type $\mathrm{III}_{\mu}$ and type $\mathrm{III}_{\mu}$-like structures in the range of $4.5-7.5 \mathrm{GHz}$ may play an important role in plasma diagnosis in the transition region.

In this paper a rare type $\mathrm{III}_{\mu}$-like structure, a V-shaped structure, observed on August 25, 1999 will be presented. The variation of reverse drift rate will be analyzed by means of the larger-scale inhomogeneity of the plasma density distribution in the solar atmosphere. The different effects of drift rate on both reverse and normal drift components as well as the parameters associated with the source region and environment are discussed. 


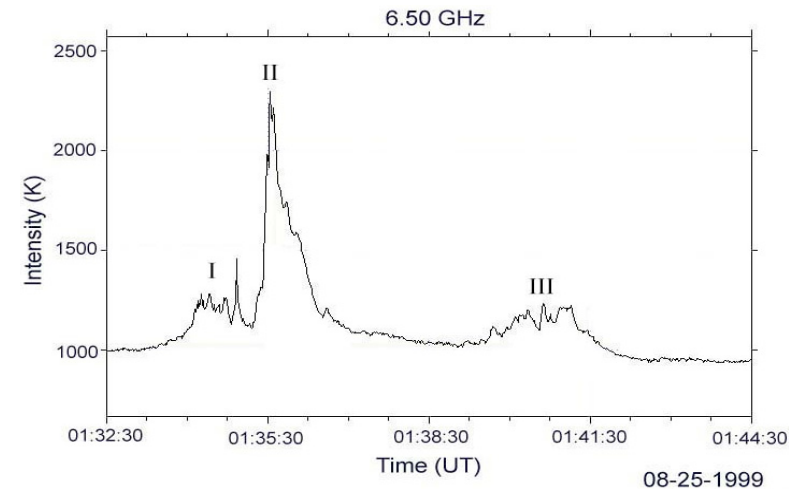

Fig. 1. Time profile of the solar radio event on August 25, 1999. It consists of three bursts, complex, strong impulse and weaker complex burst, denoted by I, II and III, respectively.

\section{Observations and data analysis}

\subsection{Observations}

The solar flare (1N/M 3.6) on August 25, 1999 occurred near the region of NOAA 8673 and 8674 . The accompanying radio burst consists of three Parts I, II and III (Fig. 1), and contains rich fine structures. To search for fine structures, especially for faint fine structures, the dynamic spectrogram corresponding to Part I was enlarged interval by interval, and its chromatic scale was also adjusted until the contrast between emissions of faint fine structure and background was large enough. Two rare V-shaped structures located in the interval of 01:34:44.396-01:34:44.960 UT and frequency range of $5.57-6.54 \mathrm{GHz}$ were detected, and their spectrogram together with the partial enlargement of the 2nd V-shaped spectrum as well as the time profile are given in Figs. 2a, b and c. As seen from Fig. 2a, the 1st V-shaped structure is a faint featureless structure and is due to an electron beam moving downward along a magnetic loop then mirroring upward. The 2nd V-shaped structure, however, is an unusual one. The 2nd one began at 01:34:44.480 UT with a starting frequency of $5.57 \mathrm{GHz}$, then drifted downward to the frequency $\sim 6.01 \mathrm{GHz}$ with a reverse drift rate $3.22 \mathrm{Ghz} / \mathrm{s}$. It then drifted downward to the turnover frequency $6.26 \mathrm{GHz}$. In this latter part, however, the reverse drift rate was about $1.53 \mathrm{GHz} / \mathrm{s}$ and was slower than that of the initial part. From the turn-over frequency, the normal drift component began with the fastest drift rate of $-3.53 \mathrm{GHz} / \mathrm{s}$ which led to an increase of the instantaneous bandwidth after the change of frequency drift. Such a feature will be discussed in Sect. 3. The variation of drift rate is a rare phenomenon.

Benz et al. (2001) stated that some artificial effects may produce a spurious symmetric pattern in the solar radio spectrogram. Therefore, a series of methods has been adopted to avoid the origin of spurious spectra during the design of the solar radio spectrometer at 4.5-7.5 GHz. More details about this technology have been reported by Yao et al. (2002). The V-shaped structure does not seem to be produced by an artificial effect due to simultaneous observations, because the simultaneous observations of the $\mathrm{V}$-shaped structures were independently obtained in the same interval and frequency range by two spectrometers located at two observatories (PMO and NAO)
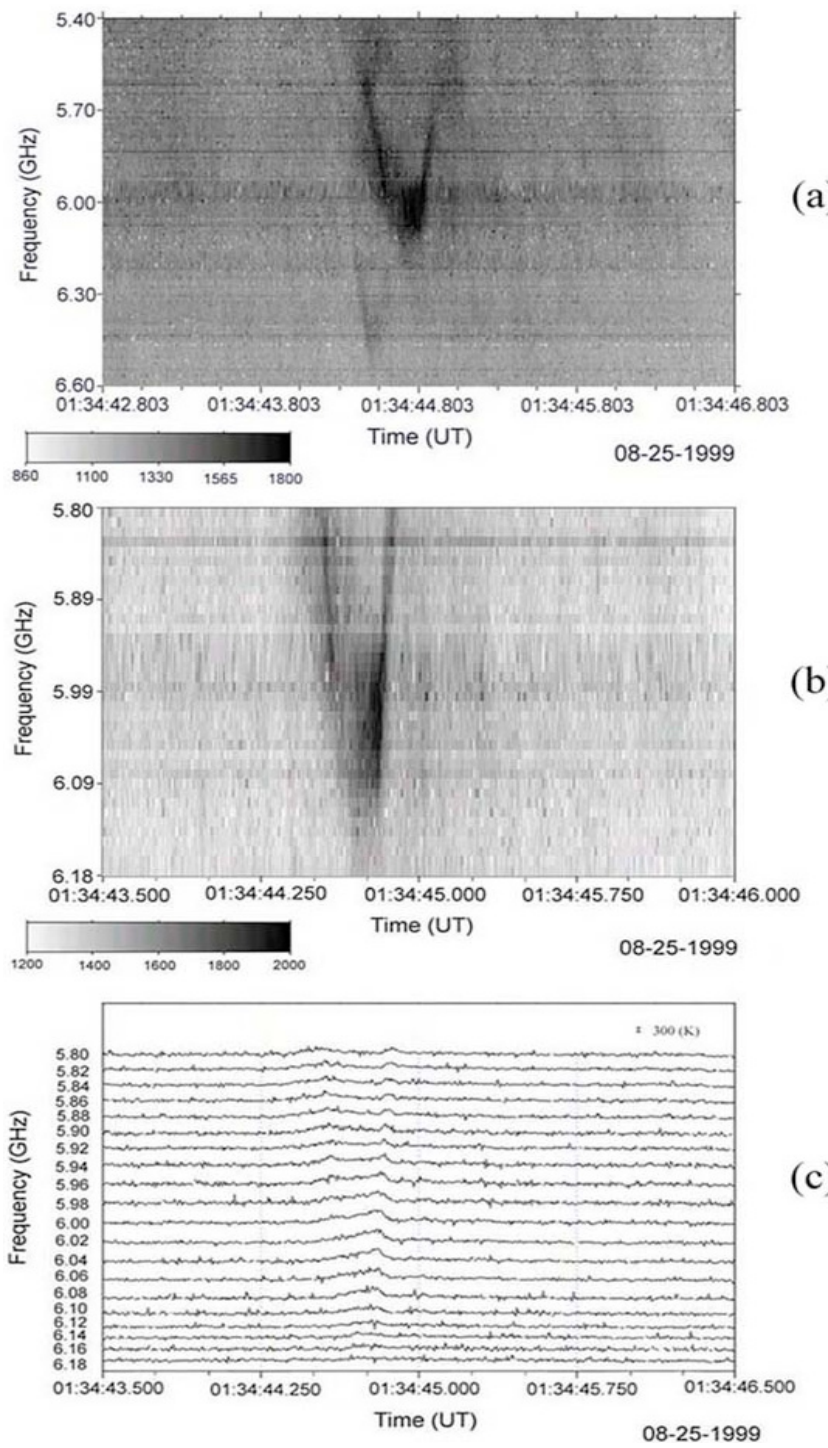

Fig. 2. Dynamic spectrogram and corresponding time profile of $\mathrm{V}$-shaped structures with time resolution of $5 \mathrm{~ms}$ and frequency resolution of $10 \mathrm{MHz}$. a) Spectrogram of two V-shaped structures. The 1 st V-shaped structure located at frequency range of $\sim 6.01-6.6 \mathrm{GHz}$ is a weak and featureless structure but the 2 nd is an unusual one with a jump of drift rate on the reverse drift component and a faster normal drift rate (compared with the reverse drift rate). b) The partial enlargement of the 2nd V-shaped structure, which could show the jump of drift rate clearly, Note that there is a strong diffusion in the frequency range of $\sim 5.90$ to $6.26 \mathrm{GHz}$ (only the higher frequency of $6.18 \mathrm{GHz}$ is shown here). c) Corresponding time profile of V-shaped structures at selected frequency. The 2 nd V-shaped structure could be seen still. Also note that in the frequency range of $\sim 5.9$ to $6.26 \mathrm{GHz}$ the peak of drift structure emission is superposed to form a continual enhancement emission, which may be caused by diffusion due to deflected electrons of lower energy.

separated by a distance of over one thousand $\mathrm{km}$. Therefore, the V-shaped structure presented here has a solar origin and hence is reliable. 


\subsection{Analysis}

Under the assumption of an isothermal atmospheric model, the electron density exponentially decreases with increasing height above photosphere i.e. $n(h)=n_{0} \mathrm{e}^{-h / \lambda}$, the frequency drift rate of type III or type III-like burst may be written as

$\frac{\mathrm{d} f}{\mathrm{~d} t}=-\frac{f v_{0} \cos \theta}{2 \lambda}$,

where $\mathrm{d} f / \mathrm{d} t, f$, the frequency drift rate and frequency, are the measurable quantities, $v_{0}$, the initial velocity of the electron beam in the $0.1-0.3 \mathrm{c}$ range, sometimes up to $0.6 \mathrm{c}$ (c-light speed), $\lambda$, the scale length to describe the density gradient, usually assumed to be $\sim 10^{10} \mathrm{~cm}$ and $\theta$, the angle between the zenith at the source location and source motion direction, which is invariable except for the source location at the mirror or near the top of the magnetic loop. As seen from (1), Eq. (1) is valid only if $v_{0}=$ constant. Therefore, $\mathrm{d} f / \mathrm{d} t$ depends on $\lambda$.

The electron density in the microwave source region, however, is two orders of magnitude higher than that in the meter or decimeter source region. Thus, energy loss of the electron beam and hence the corresponding velocity decrease via electron-electron collision must be taken into account. For an average electron of energy $E$, the energy loss rate then may be given by

$\frac{\mathrm{d} E}{\mathrm{~d} t}=-\sigma_{\mathrm{ee}} E n v$,

where $v$ is the velocity corresponding to $E, n$, the local electron density and $\sigma_{\mathrm{ee}}$, the collision cross-section for electron on electron, and $\sigma_{\mathrm{ee}}$ may be expressed as

$\sigma_{\text {ee }}=\frac{K}{E^{2}}$

where $K=55.7 \pi e^{4}$ (Brown 1971), and $e$ is the electronic charge. Then energy loss may be obtained by integration of Eq. (2)

$E=\left(E_{0}^{3 / 2}-\frac{3}{2} \sqrt{\frac{2}{m_{\mathrm{e}}}} n \Delta t\right)^{2 / 3}$,

where $m_{\mathrm{e}}$ is the electronic mass, $E$, the remaining energy after energy loss, which depends on both the initial energy $E_{0}$ and $n$ if $\Delta t$ is given. As shown by Eq. (4), under the assumption of an isothermal atmospheric model, the energy $E$ (corresponding to $v$ ) changing with time is also a slowly exponentially varying function, and hence cannot yield a jump of drift rate.

The solar atmosphere is not in equilibrium during a flare and thus an isothermal atmospheric model seems to be invalid. Based on the two-component model suggested by Aschwanden \& Benz (1986), a similar three-component model described by three exponential parts with differing scale lengths (see Fig. 3) will be assumed. The first component with a large scale length $\lambda$ represents an isothermal atmospheric model in a magnetic loop, the second and the third components with small local scale lengths $\lambda_{1}$ and $\lambda_{2}$ describe an inhomogeneous region superimposed on the first component. Such a model will apply to not only the interpretation of the change of frequency drift but also the diagnosis for plasma in the source region e.g.

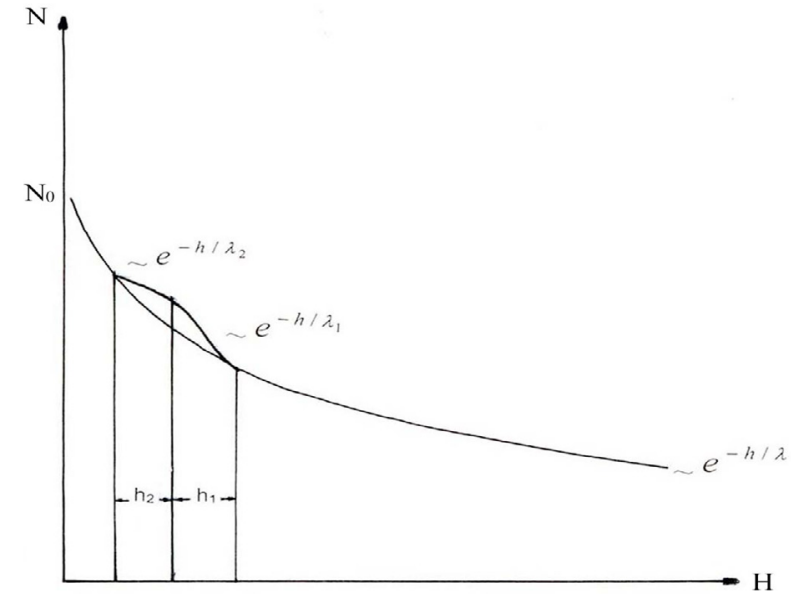

Fig. 3. Model of a three-component atmosphere. The density distribution in the magnetic loop is characterized by the scale length $\lambda$, while the inhomogeneity is described by two small local scale lengths $\lambda_{1}$ and $\lambda_{2}$.

the diagnosis for the deviation of plasma density distribution from the equilibrium solar atmospheric model as well as for the origin of the brightened knot in the soft X-ray image etc. The density in the inhomogeneous region then may be written as

$n_{i}=n_{i+1} \mathrm{e}^{-h_{i} / \lambda_{i}} \quad(i=1,2 \cdots)$,

where $n_{i}$ corresponds to the character frequency $f_{i}$ (assuming harmonic emission) of the V-shaped structure. For the sake of clearness the 2-nd V-shaped structure will also be divided into three parts according to their different drift rate denoted by numbers of 1, 2 and 3 (as shown schematically in Fig. 4). The parameters such as $f, n$ and $v$ etc. are denoted by their subscripts. For example, $f_{1}$ denotes the starting frequency, $f_{2}$, the frequency at which the reverse drift rate becomes slower, $f_{3}$, the turnover frequency and $f_{4}$, the lower-frequency of normal drift component. To estimate the energy loss at Parts 1 and 2 of V-shaped structure, Eq. (4) will be rewritten as

$E_{e i}=\left(E_{o i}^{3 / 2}-\frac{3}{2} K \sqrt{\frac{2}{m_{\mathrm{e}}}} n_{i} \Delta t_{i}\right)^{2 / 3}$,

where $E_{0 i}, E_{e i}, n_{i}$ and $\Delta t_{i}$ are the initial and remaining (i.e. at the end of part $i$ ) energy as well as the constant density and time interval in part $i$. $E_{02}$ is equivalent to $E_{e 1}$, while $E_{01}$ can be derived from the requirement that the deflection time $t_{\mathrm{D}}$ of the electron beam must exceed the total duration $t_{\mathrm{d}}$ of the type III burst. Setting $t_{\mathrm{D}}=t_{\mathrm{d}}, v_{0}$ (corresponding to $E_{0}$ ) may be determined by $t_{\mathrm{d}}$ (Benz et al. 1992)

$v_{0}=\left(\frac{10^{20} n t_{\mathrm{d}}}{3.1}\right)^{1 / 3}$,

where $t_{\mathrm{d}}$ is a measurable quantity and $n$ the density corresponding to the whole frequency range of the type III burst. For an exponential distribution of $n(h)=n_{0} \mathrm{e}^{-h / \lambda}$, however, provided $h / \lambda$ is small enough, e.g. $h / \lambda \leq 0.2$, then the local electron density may be approximately expressed by a linear function of $h / \lambda$ i.e. $n \approx n_{0}(1-h / \lambda)$, which is equivalent to its arithmetic mean $\sim n_{0}(1-h / 2 \lambda)$. Therefore, $\bar{n}_{i}$ can be expressed by 
Table 1. Parameters and drift rates for Parts 1 and 2 calculated in comparison with the observations.

\begin{tabular}{|c|c|c|c|c|c|c|c|}
\hline Part & $E(\mathrm{keV})^{\star}$ & & $v(c)^{\star}$ & & $\mathrm{h}\left(10^{9} \mathrm{~cm}\right)$ & $\lambda\left(10^{9} \mathrm{~cm}\right)$ & $\overline{\mathrm{d} f / \mathrm{d} t(\mathrm{GHz} / \mathrm{s})}$ \\
\hline & $E_{0 i} E_{e i}$ & $v_{0 i}$ & $v_{e i}$ & $\bar{v}_{i}$ & & & Cal. obs. \\
\hline 1 & $50 \quad 35$ & 0.44 & 0.37 & 0.41 & $1.655 \cos \theta$ & $10.893 \cos \theta$ & 3.243 .22 \\
\hline 2 & $35 \quad 5$ & 0.37 & 0.14 & 0.25 & $1.259 \cos \theta$ & $15.476 \cos \theta$ & $\begin{array}{ll}1.51 & 1.53\end{array}$ \\
\hline
\end{tabular}

^ Parameters with subscriptions $0 i$ or $e i$ in the 2 nd row means that the parameter in the $i$ th part is in initial or in ending state, respectively.

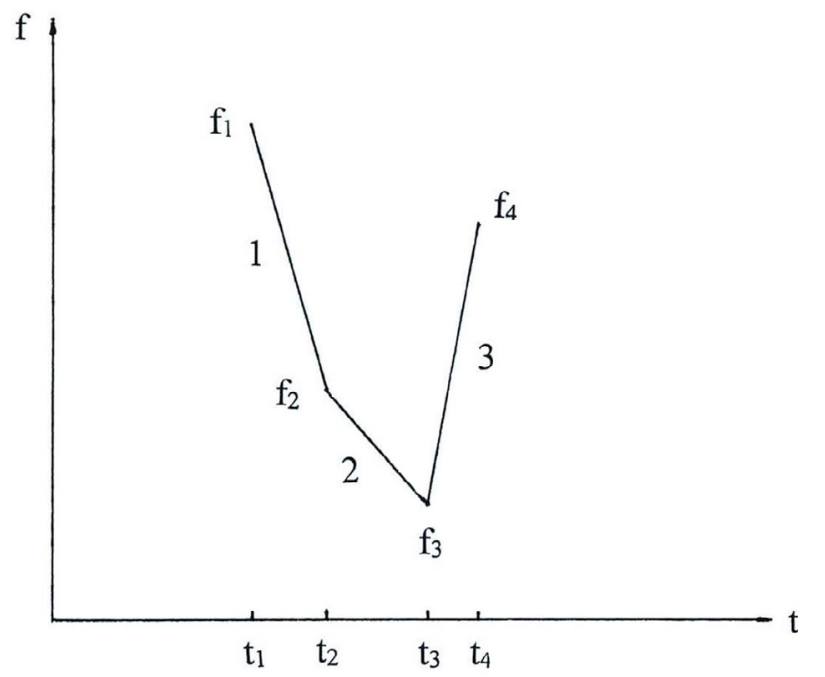

Fig. 4. An exaggerating schematic of the 2 nd V-shaped structure. The relevant parameters such as frequency $f$ as well as the different part with differing drift rate are denoted by their subscriptions $1,2,3$ and 4 .

$\left(n_{i}+n_{i+1}\right) / 2$, where $n_{i}=\left(f_{i} /\left(2 \times 8.98 \times 10^{3}\right)\right)^{2}$. Such an approximation is also applicable for our case because $h_{1} / \lambda_{1}$ and $h_{2} / \lambda_{2}$ calculated from Eq. (5) are 0.15 and 0.08. Therefore, $n_{i}$ and $n$ in Eqs. (6) and (7) will be replaced by $\bar{n}_{i}$ and $\bar{n}$. For the sake of generality, an average density $\bar{n} \approx 1 \times 10^{11} / \mathrm{cm}^{3}$ and an appropriate duration $t_{\mathrm{d}}=0.65 \mathrm{~s}$ according to the typical duration range of a type III burst given by Isliker \& Benz (1994) will be adopted in Eq. (7) for the evaluation of the initial energy $E_{0}$. By means of Eq. (6) the energy loss and the corresponding velocity (i.e. $v_{o i}, v_{e i}$, and $\bar{v}_{i}$ ) for Parts 1 and 2 then can be estimated, where $\bar{n}_{i}$ in Eq. (6) is the arithmetic mean between density $n_{i}$ and $n_{i+1}$, and can be obtained from the corresponding frequency. The quantities $\bar{n}_{1}, \Delta t_{1}$ and $\bar{n}_{2}, \Delta t_{2}$, for Parts 1 and 2 deduced from the observations are $1.042 \times 10^{11} / \mathrm{cm}^{3}, 0.136 \mathrm{~s}$ and $1.169 \times 10^{11} / \mathrm{cm}^{3}, 0.165 \mathrm{~s}$, respectively. The local scale length $\lambda_{i}$ can be determined by expression (5)

$\lambda_{i}=h_{i} / \ln \frac{n_{i+1}}{n_{i}}$,

where $h_{i}=\bar{v}_{i} \Delta t_{i} \cos \theta$. Based on the Eq. (1) the drift rate of Parts 1 and 2 can be determined by means of $\bar{v}_{i}$ and $\lambda_{i}$. The parameters and drift rates calculated are given in Table 1 . It is evident that the drift rates calculated agree well with the observations.

\section{Discussion}

The frequency drift rate of type III or type III-like structure in the same drift direction looks the same in the dynamic spectrogram because of its small time scale and narrow bandwidth. Therefore, the beam velocity $v$ and scale length $\lambda$ in Eq. (1) are usually considered as constant. However, the electron energy loss and hence the corresponding velocity variation, especially in the region with high electron density, must be considered for the estimation of electron lifetime corresponding to the deflection time $t_{\mathrm{D}}$ (usually replaced by duration $t_{\mathrm{d}}$ ). For a given $E_{0}$ and $n$, the time interval $\Delta t$ (i.e. the life-time) can be determined by Eq. (4) i.e. $\Delta t=\left(E_{0}^{3 / 2}-E^{3 / 2}\right) /\left(\frac{3}{2} K \sqrt{\frac{2}{m_{\mathrm{e}}}} n\right)$, where $E$ corresponds to $v$, and $v_{0} \geq 0.1 \mathrm{c}$ has to be satisfied. To estimate the initial velocity $v_{0}$ or initial energy $E_{0}$ of an electron beam as well as its energy loss due to electron-electron collisions, the following approaches are adopted.

Setting $t_{\mathrm{d}}=t_{\mathrm{D}}$ in Eq. (7) then the initial velocity $v_{0}$ or energy $E_{0}$ will be underestimated because $t_{\mathrm{d}}$ is shortened considerably by strong $\mathrm{f}-\mathrm{f}$ absorption (Benz et al. 1992) and hence $t_{\mathrm{d}}<t_{\mathrm{D}}$. Therefore, a mean duration of $0.65 \mathrm{~s}$ averaged over the duration of typical type III bursts given by Isliker \& Benz (1994) to determine the initial velocity $v_{0}$ or initial energy $E_{0}$ is much more reasonable than the measurable duration. On the other hand, however, the initial electron velocity $v_{0}$ in expression (1) will lead to an over-estimation of the scale length $\lambda$ if the drift rate is measurable. Therefore, in the course of derivation of the drift rates of Parts 1 and 2, an average velocity $v_{i}$ $(i=1,2)$ instead of initial velocity $v_{0}$ in Eq. (1) is suitable.

Considering an inhomogeneous region with two differing scale lengths $\lambda_{1}$ and $\lambda_{2}$, as well as different mean velocities $\bar{v}_{1}$ and $\bar{v}_{2}$, the estimated results of drift rates agree well with the observations. According to Eq. (8) $\lambda_{i}$ would be determined if $\theta$ is given. For example, $\theta=60^{\circ}$ and hence $\lambda_{1}, \lambda_{2}$ and $h_{1}, h_{2}$ are about 5.5, $7.7 \times 10^{9} \mathrm{~cm}$ and 8.3, $6.3 \times 10^{8} \mathrm{~cm}$, respectively. $\lambda_{1}<\lambda_{2}$ represents the density gradient in Part 1 which is steeper than that in Part 2. $\lambda_{1}<\lambda_{2}$ and $\bar{v}_{2}<\bar{v}_{1}$ will lead to a different drift rate of $(\mathrm{d} f / \mathrm{d} t)_{1}>(\mathrm{d} f / \mathrm{d} t)_{2}$. It is most likely that the jump in the drift rate is caused by the variations of both scale length and velocity, especially by velocity. The ratio of $\bar{v}_{2}$ to $\bar{v}_{1}$ has more effect on the jump of drift rate compared to the ratio of $\lambda_{1}$ to $\lambda_{2}$ (see Table 1 ).

The V-shaped structure is formed by an electron beam moving downward along magnetic field lines to the mirror and then upward. Consequently, the normal drift rate $\left|(\mathrm{d} f / \mathrm{d} t)_{3}\right|$ should be slower than the reverse drift rates $(\mathrm{d} f / \mathrm{d} t)_{1}$ and $(\mathrm{d} f / \mathrm{d} t)_{2}$ according to the electron velocity decrease with time due to 
energy loss. The observations, however, show that the normal drift rate $\left|(\mathrm{d} f / \mathrm{d} t)_{3}\right|$ is faster than the reverse drift rate $(\mathrm{d} f / \mathrm{d} t)_{2}$. Either a small scale length or an increasing energy may cause this. According to the drift rate $(\mathrm{d} f / \mathrm{d} t)_{3}=-3.53 \mathrm{GHz} / \mathrm{s}$ and $v_{e 2} \approx 0.1 \mathrm{c}$ given in Sect. 2.1 and Table 1 , the scale length $\lambda_{3}$ is about $10^{9} \mathrm{~cm}$ which is much smaller than $\lambda_{1}$ and $\lambda_{2}$ and is difficult to understand. Therefore, an increasing energy of the electron beam at the mirror should be taken into account. Considering an electron beam distribution with form of $\mathrm{d} F=f(E) \mathrm{d} E$, then the mean energy of the electron beam may be expressed by

$E^{\prime}=\frac{\int E f(E) \mathrm{d} E}{\int f(E) \mathrm{d} E}$,

where $E^{\prime}$ is averaged over all electrons with energy $E$. For a power law distribution, for example, $\mathrm{d} F(E)=k E^{-\delta} \mathrm{d} E$, the mean energy is

$E^{\prime}=\frac{\delta-1}{\delta-2} E_{\mathrm{c}}, \quad 2<\delta \leq 7$

where $E_{\mathrm{c}}$ is a lower energy cut-off and $E^{\prime}$ depends on $E_{\mathrm{c}}$. For a given $E^{\prime}$, therefore, the lower energy cut-off can be determined by Eq. (10). For the sake of generality, setting $\delta=4.5$ and $E^{\prime}=E_{0}=50 \mathrm{keV}$, then $E_{\mathrm{c}} \approx 35 \mathrm{keV}$. As seen from Table 1, the remaining energy $E_{e 2}$ is only about $5 \mathrm{keV}$. Therefore, electrons with energy lower than $40 \mathrm{keV}$ seem to be deflected, which is supported not only by Eq. (4) but also by observations. Based on Eq. (4), for a given density $n$ the lift-time $\Delta t$ depends only on the initial energy $E_{0}$, i.e. a lower energy $E_{0}$ corresponds to a shorter lift-time $\Delta t$. Also as seen from Fig. 2 there is a strong diffusion within the range of turn-over frequency to $\sim 5.93 \mathrm{GHz}$, which is probably caused by the deflected electrons of lower energy. Therefore, the mean energy $E^{\prime}$ of the electron beam at mirror would be $55 \mathrm{keV}$ if $E_{\mathrm{c}}=40 \mathrm{keV}$. It follows that the increasing energy is due to fewer electrons of lower energy. As a rough estimation, the normal drift rate $(\mathrm{d} f / \mathrm{d} t)_{3}=-3.96 \mathrm{GHz} / \mathrm{s}$ would be obtained by Eq. (1), where $\bar{v}_{3}$ is $\sim 0.43 \mathrm{c}$ and $\lambda_{3}$ is determined by Eq. (8). It is larger than observations, $-3.53 \mathrm{GHz} / \mathrm{s}$, but such a trend of increasing drift rate in Part 3 is acceptable.

\section{Conclusions}

Fine structures in microwave emission are still poorly known. A spectrograph with high temporal and spectral resolution is needed to study the fine structures in the microwave band. The fine structures presented here consist of a descending branch followed by an ascending one to form a V-shaped structure, which is located between 5.57 and $6.26 \mathrm{GHz}$ and lasts about $0.4 \mathrm{~s}$. The significant features of the V-shaped structure are: a distinct jump of drift rate from 3.22 to $1.53 \mathrm{GHz} / \mathrm{s}$ in the reverse drift component; a fastest drift rate of $-3.53 \mathrm{GHz} / \mathrm{s}$ in the normal drift component.

Type III or type III-like events refer to the plasma-emitting process of moving electron beams. The most significant feature of type III bursts is the frequency drift. The drift rate can be determined by both excited velocity covering the range of $\beta=0.1-0.6$ and scale length as a measure of the density gradient. As a bi-directional frequency drift event, the V-shaped structure cannot be explained by the common beam model due to different drift rates in the same drift component. The energy loss of the electron beam and the three-component solar atmospheric model with different scale lengths are proposed to explain the different drift rates. The variations of both local scale length $\lambda_{2}>\lambda_{1}$ and the mean velocity $\bar{v}_{1}>\bar{v}_{2}$, especially $\bar{v}_{1}>\bar{v}_{2}$, are responsible for the jump of drift rate in the reverse drift component. While the fastest drift rate in the normal drift component is interpreted by means of the increase of the average energy of the electron beam due to the decrease of electrons lower than $\sim 40 \mathrm{keV}$ caused by energy loss at the mirror. The local scale length $\lambda_{1}<\lambda_{2}$ means an asymmetry of local inhomogeneity, which is probably more close to the real inhomegeneity.

Acknowledgements. The authors are grateful to the referee for helpful comments. This work was supported by the National Natural Science Foundation under grants Nos. 10333030 and 10273025 and by a key Project of the Chinese Academy of Science.

\section{References}

Allaart, M. A. F., van Nieuwkoop, J., Slottje, C., \& Son daar, L. H. 1990, Sol. Phys., 130, 183

Aschwanden, M. J., \& Benz, A. O. 1986, A\&A, 158, 102

Benz, A. O., Magun, A., Stehling, W., \& Su, H. 1992, Sol. Phys., 141, 335

Benz, A. O., Messmer, P., \& Monstein, C. 2001, A\&A, 366, 326

Brown, J. C. 1972, Sol. Phys., 25, 158

Bruggmann, G., Magun, A., Benz, A. O., \& Stehling, W. 1990, A\&A, 240,506

Isliker, H., \& Benz, A. O. 1994, A\&AS, 104, 145

Melose, D. B. 1980, Aust, J. Phys., 33, 121

Stähli, M., \& Benz, A. O. 1987, A\&A, 175, 271

Suzuki, S., \& Dulk, G. A. 1985, in Solar Radiophys., ed. D. J. Mclean, \& N. R. Labrum (Cambridge Univ. Press), 289

Yao, Q. J., Xu, Z. C., Wu, H. A., \& Huang, G. L. 2002, Proc. of SOLSPA: The Second Solar Cycle and Space Weather Euroconference, 385

Zheleznyakov, V. V. 1970, Radio Emission of the Sun and Planets (Oxford: Pergamom Press) 